\title{
Li abundances and chromospheric activity of BY Dra type stars
}

\author{
Tamara V. Mishenina ${ }^{1},{ }^{2}$ Caroline Soubiran ${ }^{1}$, Valery V. Kovtyukh $^{2}$, \\ and Stanislav I. Belik ${ }^{2}$ \\ ${ }^{1}$ Université de Bordeaux - CNRS - Laboratoire d'Astrophysique de Bordeaux, \\ BP 89, 33271 Floirac Cedex, France, \\ email: caroline.soubiran@obs.u-bordeaux1.fr \\ ${ }^{2}$ Astronomical observatory, Odessa National University, \\ T.G.Shevchenko Park, Odessa 65014 Ukraine \\ email: tamar@deneb1.odessa.ua
}

\begin{abstract}
Atmospheric parameters and $\mathrm{Li}$ abundances have been determined for 162 stars observed at high resolution, high signal to noise ratio with the ELODIE echelle spectrograph (OHP, France). Among them, about 70 stars are active stars with a large fraction of BY Dra type stars. For all stars, rotational velocities were obtained with a calibration of the crosscorrelation function, effective temperatures by the line depth ratio method, surface gravities by the parallaxe method and by the ionization balance of iron. The frequency of stars with observed lithium is significantly higher in active stars than in non active stars. Among active stars, no clear correlation has been found between different indicators of activity for our sample stars, but some correlation of an index $\mathrm{R}_{H K}^{\prime}$ and $v \sin i$ is observed.
\end{abstract}

Keywords. Stars: abundances, fundamental parameters, activity

\section{Introduction}

Lithium abundances are of a particular interest, as reflecting various processes in stars and at the stellar surface and as a possible indicator of chromospheric activity. It is important also because the Sun is suspected to belong to the BY Dra type.

\section{Observations, parameters, and Li determinations}

The spectra of 162 stars were obtained using the $1.93 \mathrm{~m}$ telescope at Observatoire de Haute-Provence (OHP, France) equipped with the echelle spectrograph ELODIE (Baranne et al. 1996) which gives a resolving power of $\mathrm{R}=42000$. The spectral processing was perfomed according to Katz et al. (1998). Rotational velocities $v \sin i$ were measured with a relation calibrated by Queloz et al. (1998).

The determination of $T_{\text {eff }}, \log g,[\mathrm{Fe} / \mathrm{H}]$ was performed following Mishenina et al. (2004, 2008) and Kovtyukh et al. (2004). Li abundances $\log \mathrm{A}(\mathrm{Li})$ were determined by LTE spectral synthesis code STARSP (Tsymbal 1996). The list of lines in the region of Li I line $6707 \AA$ was taken from Mishenina \& Tsymbal (1997).

\section{Results and discussion}

Stars of BY Dra type are young stars. In their spectra the lines of lithium show different intensities not always correlating with other indicators of stellar activity. Sometimes Li lines are absent. The dependence of $\log \mathrm{A}(\mathrm{Li})$ on $T_{\text {eff }}$, is presented in Fig. 1 (the top value of an estimation of $\log \mathrm{A}(\mathrm{Li})$ are marked by symbols with arrows). 

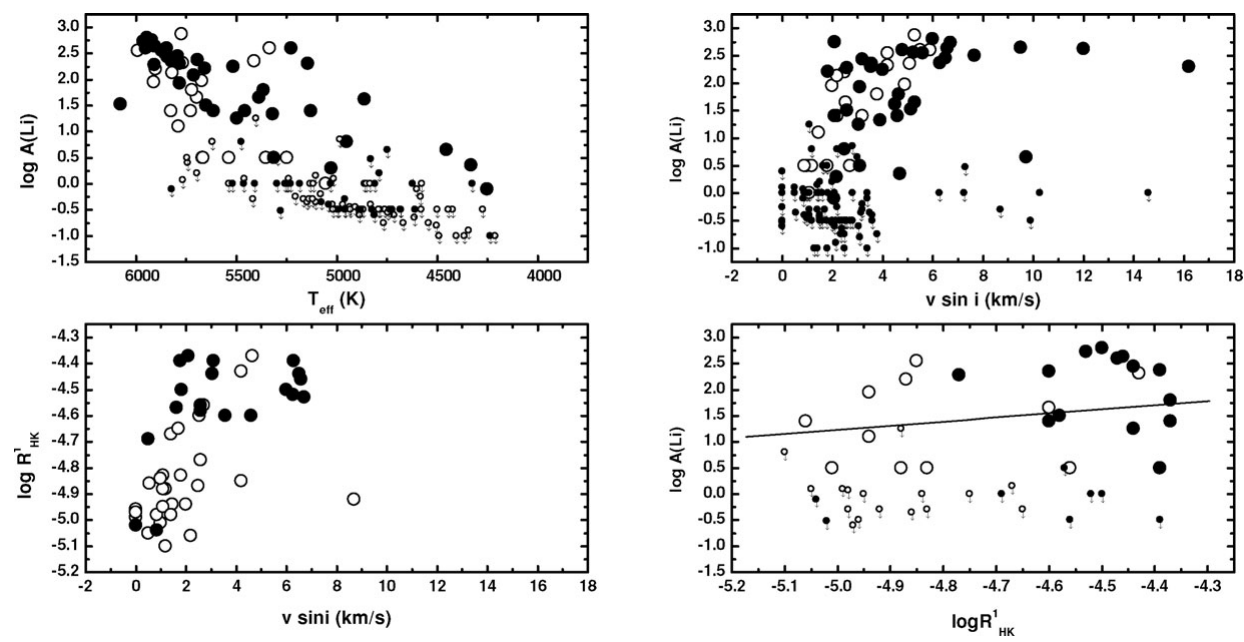

Figure 1. Connection between $\log \mathrm{A}(\mathrm{Li}), T_{\text {eff }}, v \sin i$, and $\mathrm{R}_{H K}^{\prime}$.

We have detected the lithium in 19 stars among the 91 non active dwarfs and 39 stars among the 73 stars with chromospheric activity, correspondonding to about $20 \%$ and $54 \%$, respectively. We confirm our earlier result (Mishenina et al. 2008) with almost doubled quantity of active stars. The beheavior of $\log \mathrm{A}(\mathrm{Li})$ with $v \sin i$ is similar for active and non-active stars (see Fig. 1). To search for correlations between the lithium abundance and chromospheric activity we have used as an indicator of chromospheric activity the index $\mathrm{R}_{H K}^{\prime}$ (Wright et al. 2004) for stars in a range of colours $0.4<B-V<0.9$. We have checked $\mathrm{R}_{H K}^{\prime}$ versus rotation and lithium abundance versus $\mathrm{R}_{H K}^{\prime}$ (see Fig. 1). We observe some correlation of $\mathrm{R}_{H K}^{\prime}$ and $v \sin i$, but the correlation between $\log \mathrm{A}(\mathrm{Li})$ and $\mathrm{R}_{H K}^{\prime}$ is not so obvious.

\section{Conclusions}

I. The frequency of stars with observed lithium is significantly higher in active stars than in non active stars.

II. Active stars exhibit no clear correlation between $\log \mathrm{A}(\mathrm{Li})$ and $v \sin i$ and the index $\mathrm{R}_{H K}^{\prime}$, but we observe some correlation of the index $\mathrm{R}_{H K}^{\prime}$ and $v \sin i$.

\section{References}

Baranne, A., Queloz, D., Mayor, M. et al. 1996, A\&AS, 119, 373

Katz, D., Soubiran, C., Cayrel, R. et al. 1998, A\&A, 338, 151

Kovtyukh, V. V., Soubiran, C., \& Belik, S. I. 2004, A\&A, 427, 923

Mishenina, T. V. \& Tsymbal, V. V. 1997, Pis'ma $v$ AZh, 23, 693

Mishenina, T. V., Soubiran, C., Kovtyukh, V. V., \& Korotin, S. A. 2004, A\&AA, 418, 551

Mishenina, T. V., Soubiran, C., Bienayme, O., Kovtyukh, V. V., \& Korotin, S. A. 2008, A\&A, 489,923

Queloz, D., Allain, S., Mermilliod, J.-C. et al. 1998, A\&A, 335, 183

Tsymbal, V. V. 1996, ASP Conf. Ser., 108, 198

Wright, J. T., Marcy, G. W., Buter, R. P., \& Vogt, S. S. 2004, ApJS, 152, 261 